\title{
Estudo da adsorção do corante azul de metileno em caulinita rosa
}

\author{
Paulo Vitor Brandão Leal ${ }^{1 *}$, Jeancarlo Pereira dos Anjos ${ }^{1}$ e Zuy Maria Magriotis ${ }^{1}$ \\ ${ }^{l}$ Departamento de Química; Universidade Federal de Lavras - UFLA; 37.200-000; Lavras - MG - Brasil.
}

\begin{abstract}
This work aimed to study the adsorption of blue methylene dye using as adsorbent a kind of kaolinite called(named) pink kaolinite. The adsorbent was characterized from its chemical composition, by X-ray diffraction method and surface area by BET method.

The variable parameters for the study in question were: the equilibrium time and the ratio of adsorbent mass per volume of adsorbate. It was found that the equilibrium time is less than 20 minutes and the highest adsorption occurred at 1:200 ratio, with a removal of 97\%. The isotherm adsorption was constructed from the optimized kinetics adsorption parameters. The data were linearized for the models of Langmuir and Freundlich, and Langmuir model was that better explained the results.
\end{abstract}

Key words: Adsorption, kaolinite, blue methylene

\section{INTRODUÇÃO}

Devido à preocupação com relação à preservação do meio ambiente, vem crescendo a procura por materiais de baixo custo para serem utilizados como adsorventes de corantes em meio aquoso (Pereira et al., 2008). Esta procura tem se tornado cada vez mais evidente já que diferentes indústrias (têxtil, papel, plástico, couro, dentre outras) geram uma grande quantidade de efluentes contendo resíduos de corantes que, se não forem tratados adequadamente, geram um grande problema de contaminação ambiental (Kunz et al., 2002). Dentre esses adsorventes, a caulinita apresenta-se como material promissor para remoção de cátions de meio aquoso, devido ao excesso de cargas negativas superficiais além de sua área específica (Castro, 2007; Vijayaraghavan et al., 2008).

A caulinita é de ampla ocorrência, sendo um dos mais abundantes minerais de solos e sedimentos. É derivada da alteração dos silicatos de alumínio, particularmente o feldspato, encontrada em uma mistura com feldspato nas rochas que estão sofrendo alteração, formando depósitos inferiores (Miranda-Trevino e Coles, 2003). É um argilomineral do tipo 1:1, com estrutura filossilicatada, formado pelo empilhamento regular de lâminas de tetraedros de $\mathrm{Si}$ e octaedros de $\mathrm{Al}$ (mineral dioctaedral). As unidades básicas 1:1 são ligadas por ligação de hidrogênio entre o oxigênio basal dos tetraedros e as oxidrilas da camada octaédrica (Harris et al., 2001) (Figura 1).

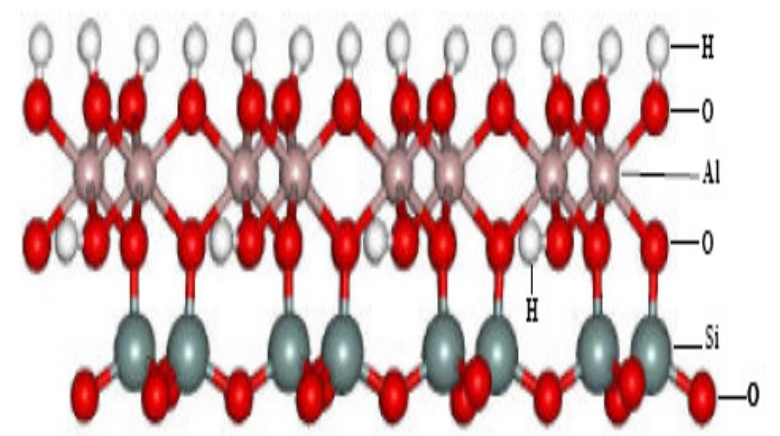

Figura 1 - Estrutura da caulinita (Adaptado de Castro, 2007).

O azul de metileno é um corante catiônico (Figura 2) utilizado em diversos campos industriais. Apesar da sua baixa toxicidade, a remoção deste de águas ou efluentes é bastante estudada devido ao fato desse corante apresentar-se como uma molécula modelo já que as bandas de absorção de suas diferentes espécies (monômeros, dímeros, agregados e espécies protonadas) são bem conhecidas e aparecem em regiões distintas do espectro (Neumann et al., 2000; Baldez et al, 2008).

$\overline{\text { Author for correspondence: pvitorufla@yahoo.com.br }}$ 


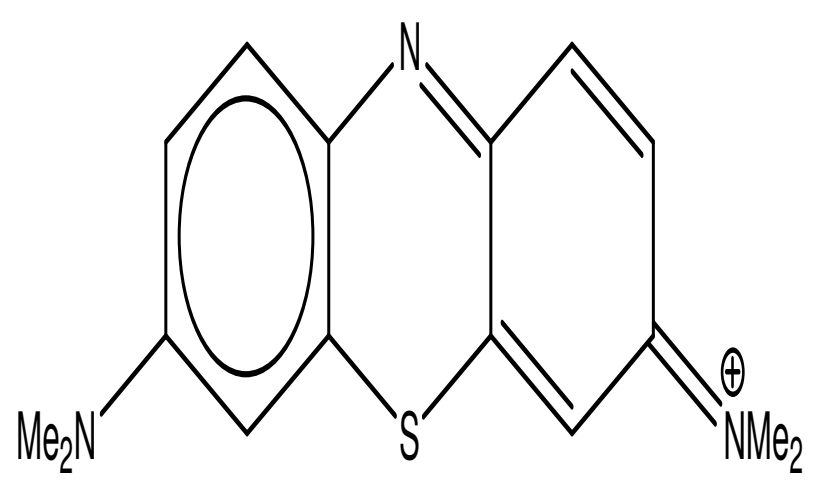

Figura 2 - Estrutura do Azul de Metileno.

Diante do exposto, o presente trabalho teve como objetivo estudar a cinética de adsorção de azul de metileno em solução aquosa, utilizando a caulinita rosa, a partir da análise do tempo de equilíbrio e da relação entre massa de adsorvente e volume de solução de adsorvato, além de propor a isoterma de adsorção.

\section{MATERIAL E MÉTODOS}

\section{Adsorvato}

A partir de uma solução estoque de azul de metileno $\left(1 \mathrm{gL}^{-1}\right)$ foram realizadas diluições para construção da curva analítica $(1,0 ; 3,0 ; 5,0 ; 7,0 \mathrm{e}$ $10,0 \mathrm{mg} \mathrm{L}^{-1}$ ) e para as cinéticas de adsorção. $\mathrm{O} \mathrm{pH}$ das soluções analisadas foi mantido próximo de 7,0 .

\section{Adsorvente}

A caulinita rosa foi cedida pela Mineradora Química e Minérios de Ijaci, Estado de Minas Gerais, Brasil, recebeu essa nomeação devido à coloração que apresentava.

A caulinita foi previamente triturada e peneirada (peneira 35 mesh Tyler) e não sofreu nenhum tratamento antes de ser utilizada nos experimento de adsorção.A composição química foi determinada por fluorescência de raios-X (Phillips CUBIX 3600). A área superficial específica foi determinada utilizando o método BET. Os resultados foram obtidos por meio de medidas de adsorção de $\mathrm{N}_{2}$ a $77 \mathrm{~K}$ utilizando o analisador ASAP 2020 (Micromeritics).

\section{Experimentos de adsorção}

Os experimentos de adsorção foram realizados em batelada, utilizando-se uma mesa agitadora (shaker) com velocidade de agitação de $100 \mathrm{rpm}$, temperatura de $25^{\circ} \mathrm{C}, 10 \mathrm{~mL}$ de solução de adsorvato e caulinita rosa como adsorvente.

Para o estudo da cinética de adsorção foram avaliados diferentes tempos de contato entre adsorvente e adsorvato $(0,15,30,60$ e 120 minutos) além da relação entre a massa de caulinita e volume de solução de azul de metileno com concentração $10 \mathrm{mg} \quad \mathrm{L}^{-1}$ (relação massa/volume: 1:100; 1:200 e 1:1000).

Foram utilizadas concentrações de azul de metileno de 2,0; 4,0; 6,0; 8,0 e $10,0 \mathrm{mgL}^{-1}$ e massa de caulinita de $0,05 \mathrm{~g}$ (equivalente a proporção de 1:200) para a construção da isoterma.

Após prévia centrifugação das amostras a 10.000 rpm por 20 minutos, em centrífuga Sigma $3 K-3$, foram realizadas leituras do sobrenadante em espectrofotômetro UV-visível SP-2000 em um comprimento de onda de $645 \mathrm{~nm}$.

\section{RESULTADOS E DISCUSSÃO Caracterização da Caulinita Rosa Área superficial}

Pela análise de BET foi obtida uma área superficial de $34.32 \mathrm{~m}^{2} / \mathrm{g}$ para a caulinita rosa, sendo este superior a valores encontrados na literatura para este material (Brady et al., 1996; Angove et al., 1997; Lee e Kim, 2002; Tekin et al., 2005; Nandi et al., 2008; Unuabonah et al., 2008).

\section{Composição química}

Por meio da caracterização da caulinita rosa pelo método de fluorescência de raio $X$ foi possível obter a composição química da caulinita rosa apresentada na Tabela 1.

Tabela 1. Composição Química da caulinita rosa

\begin{tabular}{cc}
\hline & Caulinita Rosa \\
\hline Constituinte & $\%$ \\
\hline $\mathrm{SiO}_{2}$ & 45,75 \\
$\mathrm{Al}_{2} \mathrm{O}_{3}$ & 37,41 \\
$\mathrm{Fe}_{2} \mathrm{O}_{3}$ & 2,64 \\
$\mathrm{CaO}$ & 0,00 \\
$\mathrm{MgO}$ & 0,40 \\
$\mathrm{SO}_{3}$ & 0,00 \\
$\mathrm{Na}_{2} \mathrm{O}$ & 0,08 \\
$\mathrm{~K}_{2} \mathrm{O}$ & 0,45 \\
$\mathrm{LOI}^{*}$ & 15,35 \\
\hline
\end{tabular}

*Perda por ignição 
A composição química da caulinita está de acordo com valores encontrados na literatura que afirmam ser a caulinita um filossilicato (Angove et al., 1997), constituído de tetraedros de silício ligados a octaedros de alumínio (Leja, 1982; Guerra et al., 2008).

\section{Cinética de adsorção}

No intuito de obter a melhor quantidade de adsorvente na adsorção de azul de metileno em caulinita rosa foram realizados experimentos variando-se as relações massa de adsorvente $(\mathrm{g})$ por volume de solução de azul de metileno $(\mathrm{mL})$ nas relações 1:100, 1:200 e 1:1000. As adsorções foram realizadas utilizando $\mathrm{pH}=7$, concentração inicial de azul de metileno de $10 \mathrm{mgL}^{-1}$, tempo de equilíbrio de 2 horas. As amostras foram dividas em frascos contendo $10 \mathrm{~mL}$ e foram realizadas leituras nos tempos 15, 30, 60 e 120 minutos. Os resultados da influência da massa de caulinita rosa na remoção de azul de metileno estão apresentados na Figura 3.

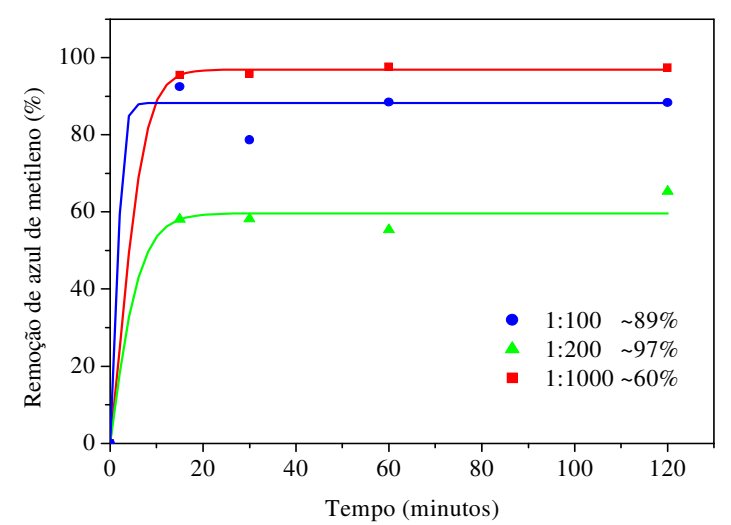

Figura 3 - Influência da quantidade de adsorvente na adsorção de azul de metileno em caulinita.

Pela análise do gráfico é possível observar que o tempo de equilíbrio de adsorção ocorreu próximo de 15 minutos para as três relações de massa:volume de caulinita e solução de azul de metileno testadas.

As porcentagens de remoção de azul de metileno por caulinita rosa foram próximas de: $60 \%$ na relação $1: 1000,89 \%$ na relação $1: 100$ e $97 \%$ na relação 1:200.

Por meio destes resultados constatou-se que a melhor relação entre adsorvente e adsorvato foi de 1:200, já que esta apresentou a maior remoção de azul de metileno após a obtenção do equilíbrio.
Para posterior construção da isoterma foi utilizada, então, essa relação de massa adsorvente e volume de solução.

De acordo com a literatura, partículas de silicato como a caulinita possuem caráter negativo com valores de $\mathrm{pH}$ acima de 2,2 (Silva, 2005). Uma vez que o pH da solução estava próximo de 7,0, possivelmente a caulinita apresentava-se carregada negativamente. Sendo o azul de metileno um corante catiônico, acredita-se que a adsorção deste em caulinita rosa ocorre por um processo de adsorção específica por meio de ligações iônicas (Neumann, 2000). Porém, alguns autores acreditam que a agregação de corantes iônicos em argilominerais não pode ser atribuída a um tipo específico de interação, já que existe a contribuição de efeitos devidos a forças do tipo Van der Waals, ligações de hidrogênio intermoleculares e interações de elétrons $\pi$ sendo, na maioria das vezes, difícil avaliar a contribuição individual de cada uma dessas interações (Neumann, 2000).

\section{Isoterma de adsorção}

Os dados de adsorção foram ajustados pelos modelos de Langmuir e Freundlich que são os mais freqüentemente usados para descrever isotermas para aplicações em tratamento de águas e efluentes.

As equações de Langmuir e de Freundlich podem ser linearizadas $\mathrm{e}$ as constantes podem ser determinadas.

A expressão linearizada de Langmuir é:

$$
\frac{C_{\mathrm{e}}}{q_{\mathrm{e}}}=\frac{1}{k V_{\mathrm{m}}}+\frac{C_{\mathrm{e}}}{V_{\mathrm{m}}}
$$

Já a isoterma de Freundlich é utilizada para a modelagem da adsorção de superfícies heterogêneas. A equação linearizada desta isoterma pode ser explicada pela equação (2):

$$
\log q_{\mathrm{e}}=\log K_{\mathrm{f}}+\frac{1}{n} \log C_{\mathrm{e}}
$$

Os resultados das linearizações das isotermas de Langmuir e Freundlich adsorção de azul de metileno em caulinta rosa estão apresentadas na Tabela 2. 
Tabela 2. Linearização dos dados das isotermas para Langmuir e Freundlich

\begin{tabular}{lcccccc}
\hline & \multicolumn{3}{c}{ Langmuir } & \multicolumn{2}{c}{ Freundlich } \\
\cline { 2 - 7 } & $\mathrm{V}_{\mathrm{m}}$ & $\mathrm{K}$ & $\mathrm{r}^{2}$ & $\mathrm{~K}_{\mathrm{f}}$ & $\mathrm{n}$ & $\mathrm{r}^{2}$ \\
\hline Caulinita Rosa & 14,286 & 0,084 & 0,995 & 0 & 1,887 & 0,698 \\
\hline
\end{tabular}

A partir dos resultados obtidos pelas isotermas foi possível observar que os dados se ajustaram melhor ao modelo de Langmuir, já que para este foi obtido um melhor coeficiente de correlação $\left(\mathrm{R}^{2}\right.$ $=0,9950)$, mostrando a melhor linearização dos dados. Esse fato está de acordo com alguns estudos encontrados na literatura, que avaliaram a adsorção de adsorvatos em diferentes tipos de argilas (Turak, 2004; Baskaralingam, 2007; Nandi et al., 2008; Roulia e Vassiliadis, 2008).

A Figura 4 mostra a isoterma de adsorção do azul de metileno em caulinita rosa onde se pode constatar uma capacidade máxima de adsorção de, aproximadamente, $1,2 \mathrm{mg} \mathrm{g}^{-1}$ do material estudado. Este resultado mostra-se relativamente inferior quando comparado à outros estudos de adsorção (Turak, 2004; Baskaralingam, 2007; Roulia e Vassiliadis, 2008; Bharttachaya e Gupta, 2008; Unuabonah et al., 2008). Estudos de Pereira et al. (2008), os quais avaliaram a eficiência de adsorção dos corantes azul de metileno e vermelho reativo em diferentes carvões ativados obtidos de rejeitos de café, apresentaram máximos de adsorção de 14 a $71 \mathrm{mg} \mathrm{g}^{-1}$, aproximadamente, para a adsorção do azul de metileno nos diferentes carvões testados (Pereira e Oliveira, 2008).

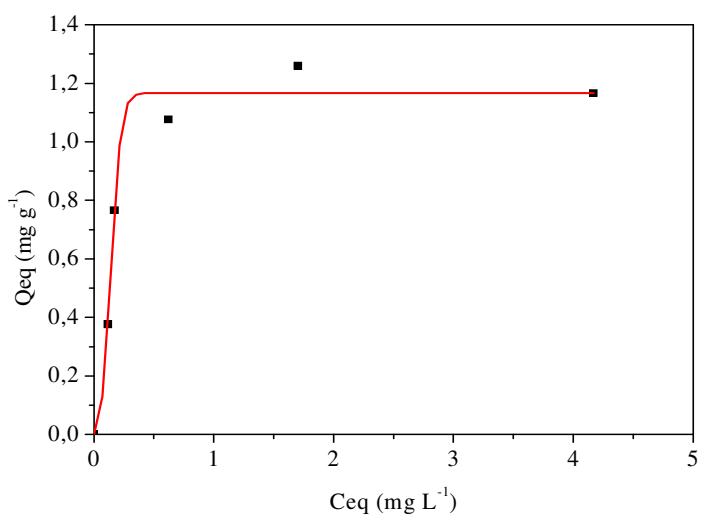

Figura 4 - Isoterma de adsorção de azul de metileno em caulinita rosa.

\section{CONCLUSÕES}

Pelos resultados obtidos para o estudo da cinética de adsorção do corante azul de metileno em caulinita rosa, pode-se inferir que o tempo de equilíbrio mostrou-se relativamente baixo $(t=15$ min.), sendo esta uma característica desejável na obtenção de novos materiais adsorventes. A melhor relação entre massa de adsorvente rosa e volume de solução de adsorvato obtida, nas condições experimentais especificadas neste trabalho, foi de 1:200.

$\mathrm{O}$ modelo de isoterma mais adequado para o estudo da adsorção de azul de metileno em caulinita rosa foi o de Langmuir, já que os dados apresentaram melhor linearização.

Com relação à capacidade máxima de adsorção do corante utilizando a caulinita rosa, pode-se inferir

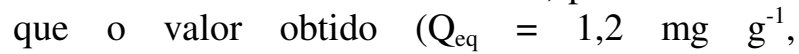
aproximadamente) mostrou-se relativamente baixo quando comparado a outros estudos de adsorção, apesar de ser satisfatório por não apresentar grande discrepância em relação aos dados da literatura.

Pela caracterização da caulinita por meio da determinação de sua área superficial e pelos resultados da cinética de adsorção, acredita-se que a caulinita rosa seja um promissor adsorvente para a remoção de azul de metileno em meio aquoso.

\section{RESUMO}

Este trabalho teve como objetivo estudar a adsorção do corante azul de metileno usando como

adsorvente uma espécie de caulinita chamada caulinita rosa. A caulinita foi caracterizada a partir da sua composição química, pelo método de difratometria de raio-X e área superficial, pelo método de BET.Os parâmetros variáveis para o estudo em questão foram: o tempo de equilíbrio e a relação entre massa de adsorvente por volume de adsorvato. Verificou-se que o tempo de equilíbrio é menor do que 20 minutos e o máximo de adsorção ocorreu na razão de 1:200, com uma remoção de $97 \%$. A isoterma de adsorção foi construída a partir de parâmetros otimizados da cinética de adsorção. Os dados foram linearizados para os modelos de Langmuir e Freundlich, e modelo de Langmuir foi que melhor explicou os resultados. Palavras-chave: Adsorção, azul de metileno, caulinita 


\section{REFERÊNCIAS}

Angove, M. J.; Johnson, B. B.; Wells, J. D. (1997), Adsorption of Cadimium (II) on kaolinite. Colloids and Surfaces A, 126, 137-147.

Baldez, E. E.; Robaina, N. F.; Cassella, R. J.(2008), Employment polyurethane foam for the adsorption of methylene blue in aqueous medium. Journal of Hazardous Materials, 159, 580-586.

Baskaralingam, P.; Pulikesi, M.; Ramamurthi, V.; Sivanesan, S. (2007), Modified hectorites and adsorption studies of a reactive dye. Applied Clay Scince, 37, 207-214.

Bharttachayya, K. G. and Gupta, S. S. (2008), Kaolinite and montmorillonite as adsorbents for $\mathrm{Fe}(\mathrm{III}), \quad \mathrm{Co}$ (II) and $\mathrm{Ni}(\mathrm{II})$ in aqueous medium.Applied Clay Science, 41, 1-9.

Brady, V. P.; Cygan, T. R.; Nagy, L. K. (1996), Molecular Controls on Kaolinite Surface Charge. Journal of Colloid Interface Scince, 183, 356-364.

Castro, E. A. S. and Martins, J. B. L. (2005), Theoretical study of benzene interaction on kaolinite. Journal of Computer-Aided Materials Design, 12, 121-129.

Guerra D. L.; Souza, J. A.; Airoldi, C.; Viana, R. R. (2008), Avaliação da eficiência de caulinita intercalada com dimetilsulfóxido em adsorção com $\mathrm{Zn}$ (II) em meio aquoso - cinética do processo de adsorção. Cerâmica, 54, 273-279.

Harris, R. G.; Wells, J. D.; Johnson, B. B. (2001), Selective adsorption of dyes and other organic molecules to kaolinite and oxide surfaces. Colloids and Surface A, 180, 131-140.

Kunz, A.; Peralta-Zamora, P.; Moraes, S. G.; Durán, N. (2002), Novas tendências no tratamento de efluentes têxteis. Química Nova, 25, 78-82.

Lee,Y. S. and Kim, J. S. (2002), Adsorption of naphthalene by HDTMA modified kaolinite and halloysite. Applied Clay Science, 22, 55-63.

Leja, J. Surface chemistry of froth flotation. New York: Plenum Press, 1982.

Miranda-Trevino, J. C. and Coles, C. A. (2003), Kaolinite properties, structure and influence of metal retention on $\mathrm{pH}$. Applied Clay Scince, 23, 133-139.

Nandi, B. K.; Goswami, A.; Purkait, M. K. (2008), Removal of cationic dyes from aqueous solutions by kaolin: Kinetic and equilibrium studies. Applied Clay Scince, 42, 583-591.

Neumann, M. G; Gessner, F; Cione A. P. P; Sartori A. R; Cavalheiro, S. C. C. (2000), Interações entre corantes e argilas em suspensão aquosa. Química Nova, 23, 818-824.

Pereira, E.; Oliveira, L. C. A.; Vallone, A.; Sapag, K. (2008), Preparação de carvão ativado em baixas temperaturas de carbonização a partir de rejeitos de café: utilização de $\mathrm{FeCl}_{3}$ como agente ativante. Química Nova, 31, 1296-1300.

Roulia, M. and Vassiliadis, A. A. (2008), Sorption characterization of a cationic dye retained by clays and perlite Microporus and Mesoporus Materials, 116, $732-740$.

Silva, F. R. A. Avaliação de processosde adsorção de metais pesados: um estudo experimental com propostas de utilização de subprodutos. Dissertação (Mestrado em Sistemas de Gestão) Universidade Federal Fluminense, 2005.

Tekin, N.; Demirbas, O.; Alkan, M. (2005), Adsorption of cationic polyacrylamide onto kaolinite. Microporous Mesoporous Materials, 85, 340-350.

Turak, F.; Ozgur, M.U.; Afsar, H. (2004), Use of natural and activated clay to remove methylene blue from aqueous solutions. In: $4^{\text {th }} A A C D$ Congress, Adnan Menderes University.

Unuabonah, E. I.; Adebowale, K. O.; Dawodu, F. A. (2008), Equilibrium, kinetic and sorber design studies on the adsorption of Aniline blue dye by sodium tetraborate-modified Kaolinite clay adsorbent. Journal of Hazardous Materials, 157, 397-409.

Vijayaraghavan, K.; Won, S. W.; Mao, J.; Yun, Y. (2008), Chemical modification of Corynebacterium glutamicum to improve methylene blue biosorption. Journal of Chemical Engineering, 145, 1-6. 